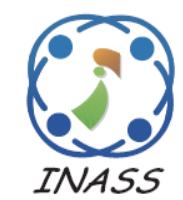

\author{
International Journal of \\ Intelligent Engineering \& Systems
}

http://www.inass.org/

\title{
Broadband GaAs FET Power Amplifier for L and S Bands Applications
}

\author{
Mohamed Ribate $^{1 *} \quad$ Jamal Zbitou $^{1} \quad$ Rachid Mandy $^{1} \quad$ Ahmed Erkik $^{1} \quad$ Mohamed Latrach $^{2}$ \\ ${ }^{1}$ Mechanical, Energy, Electronics and Telecommunication laboratory, Sciences and Technologies Faculty, \\ Hassan $1^{\text {st }}$ University, Settat, B.O Box 577, Morocco \\ ${ }^{2}$ Microwave Group ESEO Angers, French \\ * Corresponding author's Email: ribate.mohamed@gmail.com
}

\begin{abstract}
This paper proposes a Broadband Power Amplifier (BPA) for L and S bands applications based on ATF13786 transistor, using GaAs process. To improve the broadband performance, an approximate transformation of previously designed lumped elements into transmission lines, and a multi-section quarter wave impedance transformer are used. With neatly design of the broadband networks and bias circuit, a maximum gain of $14.89 \mathrm{~dB}$ is achieved across $1.1 \mathrm{GHz} \sim 3 \mathrm{GHz}$. At $2 \mathrm{GHz}$, the simulated large signal results demonstrate that the designed BPA achieves a saturated output power of $17 \mathrm{dBm}$, with $1-\mathrm{dB}$ compression point at $4 \mathrm{dBm}$ input power level, and a PAE of $20 \%$. For the whole bandwidth, the input return loss and output return loss are below than $-10 \mathrm{~dB}$. The maximum value achieved by the reverse transmission is $-20 \mathrm{~dB}$ over the operation frequency ranges. Considering the broad frequency coverage, the performance of the proposed design compares favorably with the state-of-art.
\end{abstract}

Keywords: Broadband power amplifier (BPA), GaAs, L and S bands, Microstrip line, Single DC supplies.

\section{Introduction}

Among electronic circuits, broadband signal amplification is one of the most important microwave and radiofrequency (RF) circuit functions [1]. The spectrum around us is full of transmitted signals waiting to be received. Each of these signals was transmitted by some sort of power amplifier [2].

Thereby, every circuit designer is likely to face the challenge of designing a microwave or radiofrequency transmitter, and inherently a Broadband Power Amplifier (BPA) for one of the following bands (defined by IEEE Standard 5211984) [3]: $\mathrm{L}-\mathrm{S}-\mathrm{C}-\mathrm{X}$ or $\mathrm{Ku}$ bands [4] operating at Ultra High Frequency (UHF) and Supra High Frequency (SHF) from $30 \mathrm{~cm}$ of wavelength, to $1.7 \mathrm{~cm}(1 \mathrm{GHz}$ to $18 \mathrm{GHz})$ [5].

However, BPA applications span a wide range of areas, among which, Cellular Communication - be it a base station transceiver of Global System Mobile (GSM), Universal Mobile Telecommunications System (UMTS) or Long Term Evolution (LTE) radars [6], avionics, electronic warfare, digital television broadcast, heating, and medical microwave imaging represent just a few examples [7, 8].

This miscellaneous and evolving broadband power amplifier landscape dictates the strategy for the design, optimization and fabrication of multiple generations of RF power devices.

The active device - RF power transistor - must satisfy a broad and often conflicting set of application requirements, including but not limited to stability, output power, bandwidth, gain, linearity, efficiency and cost effectiveness [9].

The amplifier topology and architecture has also evolved to fit to the ever changing system requirements, most recently with the widespread adoption of Doherty configuration to increase the back-off efficiency in linear applications [10 - 12], but the performance will be limited when the bandwidth requirement is larger than octave.

To date, many design and implementation of broadband power amplifier have been proposed in the literature using balanced structures, distributed configurations [13 - 15] and several matching techniques such as Real Frequency Technique (RFT), 
resistive matching circuits and reactive filter synthesis [16]. The resistive matching techniques and reactive filter synthesis cannot obtain good return loss over a wide frequency range and both have some limitation in extending the bandwidth of the power amplifier. Conversely, the RFT has clear advantages in terms of its return loss and bandwidth.

In this work, we adopt the Real Frequency Technique and other broadband matching techniques in the design of the input and output matching networks. The RFT is probably the best circuit synthesis method available for the design of broadband power amplifiers. It is being applicable to all matching problems, as it does not need a predefined equalizer topology [24]. In addition, comparing this technique with similar contemporary state of art broadband matching techniques, the RFT shows good return loss and a wider bandwidth.

In this paper we present the design of a Broadband Power Amplifier (BPA) over a $2900 \mathrm{MHz}$ bandwidth, from $1.1 \mathrm{GHz}$ to $3 \mathrm{GHz}$, which cover the mainstream communication standards running in $\mathrm{L}$ and $\mathrm{S}$ bands. Furthermore, the proposed BPA reaches a maximum gain of $14.89 \mathrm{~dB}$, and a saturated output power of $17.14 \mathrm{dBm}$ with $20 \%$ of maximum PAE, while the design achieves a simple configuration and an excellent matching performance over a broad frequency range.

This paper is organized as follows. In section 2, the deployed broadband matching techniques and biasing networks are presented. The simulation results are given in section 3. The proposed BPA layout is given in section 4 and the concluding remarks are summarized in section 5 .

\section{Microwave circuit design}

Whether characterized as broadband, narrowband, low noise, high power or otherwise, all electronic amplifiers have common characteristics of providing finite positive Power Gain at the frequency or range of frequencies, of interest [17].

Broadband Power Amplifier design requires accurate RF active device modelling, efficient impedance matching, stability throughout operation, and ease of practical implementation. The feature of a broadband power amplifier design is evaluated by its ability to reach maximum power gain across the required frequency bandwidth, under stable operating conditions, with minimum amplifier stages, and the requirement for the high efficiency or linearity can be considered where it is needed. We note, for stable operation, it is necessary to evaluate the operation frequency domains in which the active device may be potentially unstable [18].

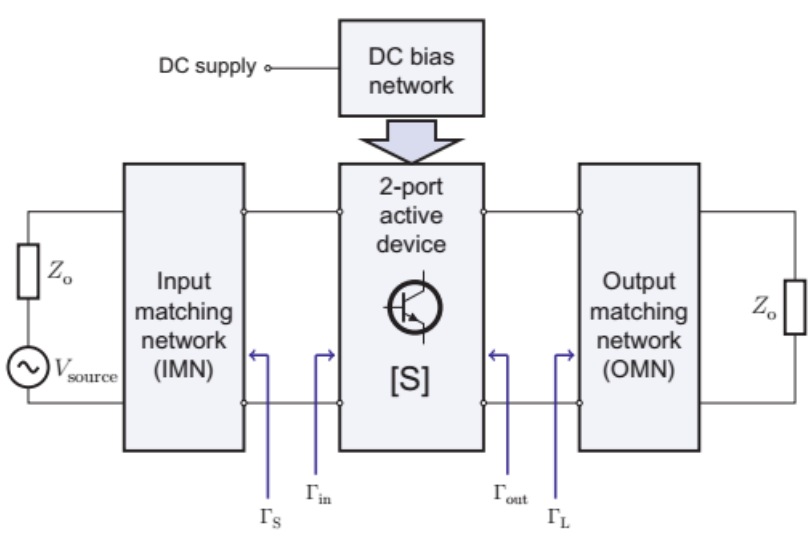

Figure. 1 Single active device with input and output matching network

Fig. 1 shows the basic block schematic for a single stage power amplifier circuit which includes an active device, an Input Matching Network (IMN) to match with the source impedance, an Output Matching Network (OMN) to match with the load impedance, and a bias network to supply DC supply power to the transistor.

\subsection{Broadband impedance matching techniques}

Impedance matching or impedance transformation is the key part of a Broadband power amplifier design. Generally, when there is a mismatch between the input (source) and the output (load), the RF power available from the source is not delivered to the load and this makes a loss of power. This means that, a portion of electrical signal will be reflected at the interface between sections with different impedances, when this signal propagates in the circuit. Thus, the impedance matching is very necessary to provide a maximum transfer of RF power from a source to an active device, from an active device to a load, or between devices.

There are various techniques of impedance matching that can be used to match two given impedances such as: lumped elements, transmission lines and multi-section Quarter Wave impedance transformers. In addition, both single-stub and double-stub as well as a quarter-wavelength transmission line impedance matching can be used. The main factors to be considered in the chosen of a matching technique are: bandwidth, complexity, frequency response and ease of implementation.

At high frequency, excepting an MMIC (Monolithic Microwave Integrated Circuit), it is generally difficult to implement the lumped elements on a PCB. However, a matching network that uses transmission lines is easy to implement on a PCB substrate. 


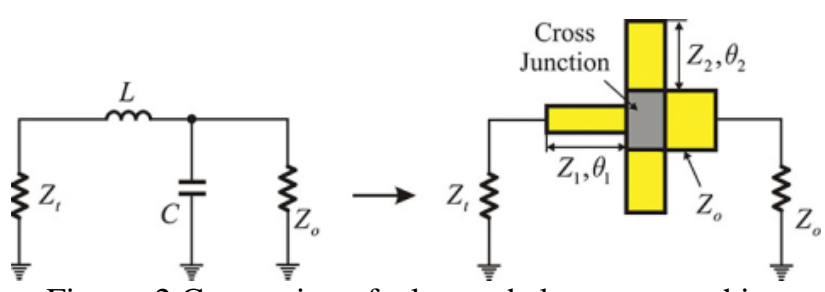

Figure. 2 Conversion of a lumped elements matching circuit to a transmission lines matching circuit

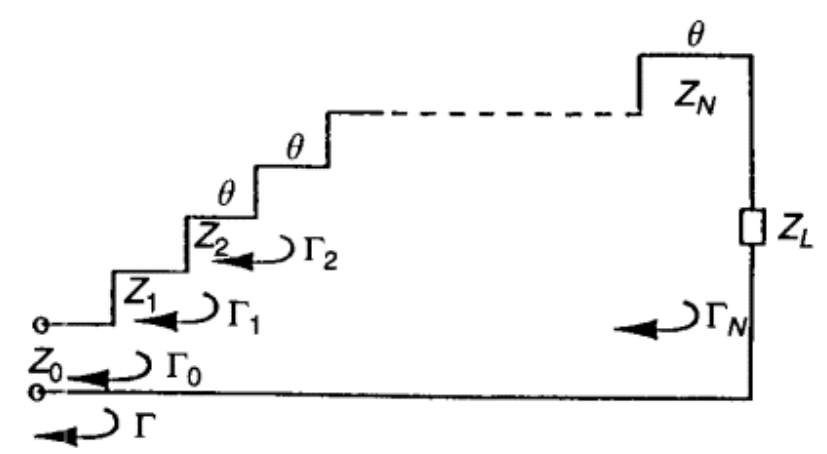

Figure.3 Multi-Section Quarter Wave Transformer ( $\mathrm{N}=$ Number of sections)

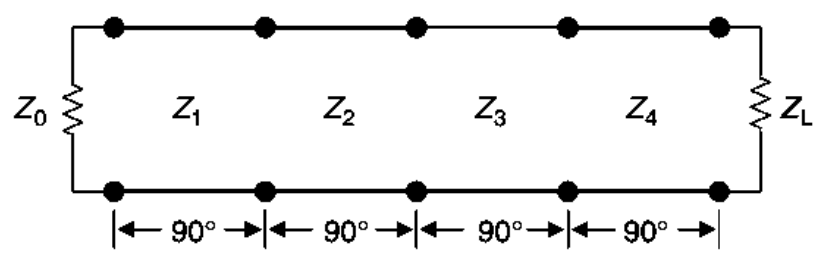

Figure. 4 Four sections binomial transformer

In this work, more than technique is used to improve the broadband performance in input and output matching networks. The first one, is an approximate transformation of a formerly designed lumped elements matching circuit, into the transmission lines matching circuit. This approximation can be done by swapping the lumped elements by the transmission lines.

Fig. 2 shows the transmission lines circuit that can replace the designed lumped elements matching circuit. The series inductor is transformed into the transmission line with characteristic impedance $Z_{1}$ and length $\theta_{1}$.

The shunt capacitor is transformed into the two parallel-connected transmission line stubs with characteristic impedance $Z_{2}$, and length $\theta_{2}$. The values of $Z_{1}, \theta_{1}, Z_{2}$, and $\theta_{2}$ can be approximately calculated by using the following equations [19]:

$$
\begin{aligned}
& X_{1}=Z_{1} \tan \left(\theta_{1}\right) \approx \omega L \\
& X_{2}=\frac{Z_{2}}{2 \tan \left(\theta_{2}\right)} \approx \frac{1}{\omega C}
\end{aligned}
$$

Form Eq. (1), the variables are $Z_{1}$ et $\theta_{1}$, thus, there are two degrees of freedom in implementing the value of an inductor. The same for the Eq. (2).

The second technique used in this work is the Multi-Section Quarter Wave impedance transformer.

Fig. 3 shows the schematic of a Multi-Section Quarter Wave Transformer in which the load impedance $\mathrm{Z}_{\mathrm{L}}$ is transformed to $\mathrm{Z}_{0}\left(\mathrm{Z}_{0}\right.$ is the characteristic impedance of the feed line). The transformer consists of discrete sections of transmission lines having different characteristic impedances, but the same electrical length $\beta l=\theta$. The electrical length will be a quarter wave length at the band center frequency $\mathrm{f}_{0}$. The transformer is symmetrical, thus the reflection coefficients at junctions of the sections are related as $\left|\Gamma_{0}\right|=$ $\left|\Gamma_{N}\right|,\left|\Gamma_{1}\right|=\left|\Gamma_{N-1}\right|, \ldots$

There are two widely known Multi-Section Quarter wave transformers: Binomial and Chebyshev.

A schematic diagram of a binomial transformer is illustrated in Fig. 4. In the case of transmission lines sections, $\theta=\pi f / 2 f_{0}$, and consequently the fractional bandwidth is given by:

$$
\begin{aligned}
\frac{\Delta f}{f_{0}} & =\frac{2\left(f_{0}-f_{m}\right)}{f_{0}} \\
& =2-\frac{\pi}{4} \cos ^{-1}\left|\frac{2 \rho_{m}}{\ln \left(\frac{Z_{L}}{Z_{0}}\right)}\right|^{1 / N}
\end{aligned}
$$

$\mathrm{N}$ is the number of sections and $\rho_{m}$ is the tolerable reflection coefficient in the passband.

The impedances of the sections can be approximately calculated by using the following equations [20]:

$$
\begin{gathered}
\ln \frac{Z_{n+1}}{Z_{n}}=2 \rho_{n}=2^{-N} C_{n}^{N} \ln \frac{Z_{L}}{Z_{0}} \\
C_{n}^{N}=\frac{N !}{(N-n) ! n !}
\end{gathered}
$$

Where $Z_{n}$ and $Z_{n+1}$ are the impedances of the $n$th and $(n+1)$ th sections, respectively, $\rho_{n}$ is the reflection coefficient at the junction between $Z_{n}$ and $Z_{n+1}$, and $C_{n}^{N}$ are the binomial coefficients. In the case of a two-section binomial transformer:

$$
\begin{aligned}
& Z_{1}=Z_{L}^{1 / 4} Z_{0}^{3 / 4} \\
& Z_{2}=Z_{L}^{3 / 4} Z_{0}^{1 / 4}
\end{aligned}
$$




\subsection{Biasing network}

Biasing networks plays an important role in the design of an RF power amplifier. They supply the efficient excitation means for active devices. The biasing parameters can change with the active device technology, but the design basics for biasing methods are basically the same.

Biasing of transistors includes two parts: the chosen of bias Q-point (Quiescent Point) for optimum device performance in terms of stability, gain, PAE, output power, linearity, noise figure, RF choking.... and the biasing networks.

Basically, a biasing network comprise a DC block and a RF chock. The aim of RF chock is to have very high impedance at the operating frequencies to prevent the RF energy from seep through the biasing network. Generally, a DC block can be either a capacitor or 3-db backward-wave coupler, and should be present short circuit at the operation frequencies. The value of the capacitor should be selected such that $w C=2 \pi f C$ is very large.

Consequently, $Z_{c}=-j\left(\frac{1}{w C}\right)$ is very small and the signal can pass through the capacitor with very weak reflection.

At microwave frequencies, the RF chock is generally realized by using a high impedance $\lambda / 4$ line terminated by an open circuited quarter wave low impedance line. The configuration is shown in Fig. 5.a. In order to increase the bandwidth, the low impedance line section can be replaced with a radial line section as shown in Fig. 5.b, and this configuration can provide a small size and better bandwidth than a quarter wave open circuited line section.

\subsection{The proposed broadband power amplifier}

By using the concepts mentioned above, the proposed BPA is shown in Fig. 6.

The design of this broadband power amplifier is based on commercial transistor ATF786, which is a Gallium Arsenide Schottky barrier-gate Field Effect Transistor.

In the design procedure, the bias condition (known as DC Q-point) for the active device should be carefully selected, because it determines the basic performance in terms of stability, output power, gain and PAE of the whole power amplifier.

The employed bias network is shown in Fig. 7. The proposed power amplifier is biased with single supply voltage $\mathrm{VCC}$ of $+3.5 \mathrm{~V}$. According to the broadband requirement, the RF chock is realized by using a radial stub followed by a high impedance $\lambda / 4$ transmission line. This combination allows to preventing the RF energy from entering the power supply and increases the bandwidth.

As shown in Fig. 8 and Fig. 9, the input matching and the output matching networks have been designed as simple as possible to reduce both the layout consumption and the gain loss. The design of both matching networks depends on the range of frequencies wherein this broadband power amplifier need to be designed.

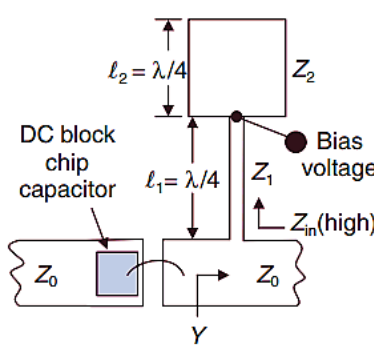

(a)

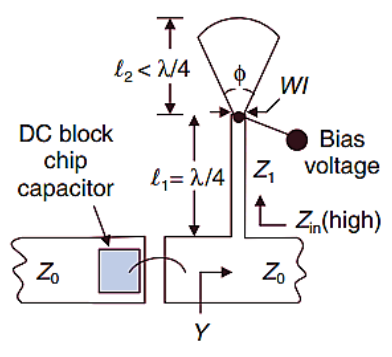

(b)
Figure. 5 Simplified microwave biasing circuits: (a) MIM capacitor DC block and two $\lambda / 4$ transformers and (b) MIM capacitor DC block and $\lambda / 4$ transformer in series with a radial line stub

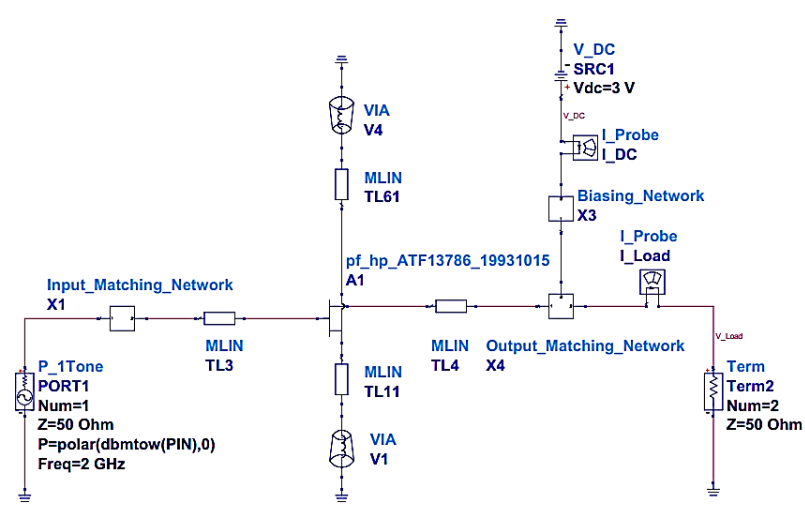

Figure. 6 The proposed single stage broadband power amplifier schematic

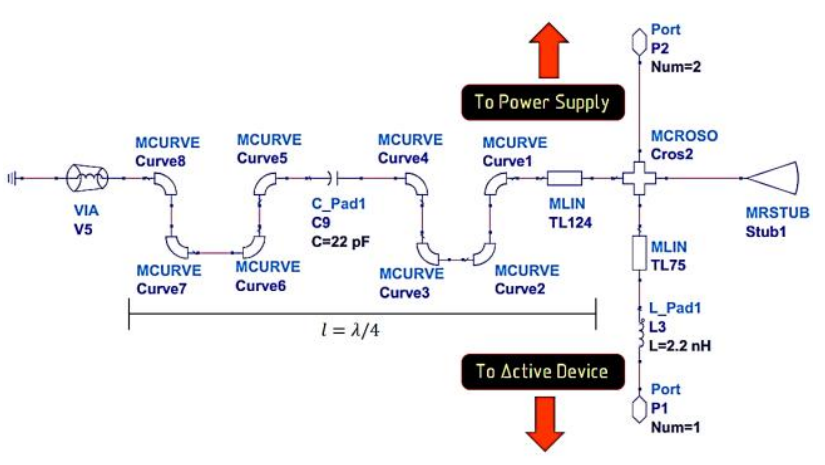

Figure. 7 Biasing network 


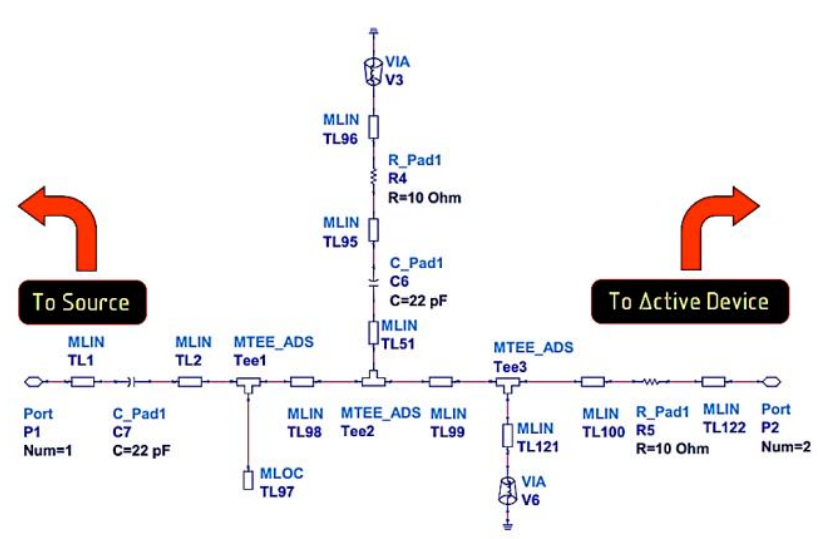

Figure. 8 Input matching network

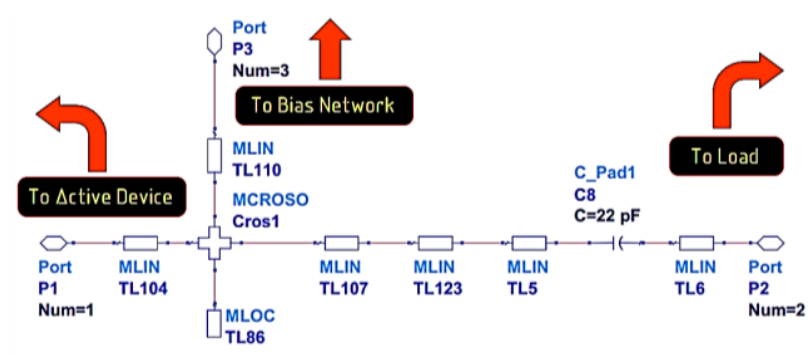

Figure. 9 Output matching network

\section{Simulation results and discuss}

\subsection{Stability analysis}

According to the S-parameters approach to the stability analysis of the power amplifier, unconditional stability is assured if the following equations are simultaneously satisfied:

$$
\left\{\begin{array}{l}
k=\frac{1+|\Delta|^{2}-\left|S_{11}\right|^{2}-\left|S_{22}\right|^{2}}{2\left|S_{12} S_{21}\right|}>1 \\
B_{1}=1+\left|S_{11}\right|^{2}-\left|S_{22}\right|^{2}-|\Delta|^{2}>0
\end{array}\right.
$$

Where :

$$
\Delta=S S_{11} S_{22}-S_{12} S_{21}
$$

$\mathrm{k}$ : Rollett stability factor.

$\mathrm{B}_{1}$ : Bodway stability factor.

Figs. 10 and 11 show the simulated $\mathrm{k}$ and $\mathrm{B}_{1}$ across $1.1 \mathrm{GHz} \sim 3 \mathrm{GHz}$. The Rollett and the Bodway stability factors achieves a minimum value of 1.42 and 0.86 respectively.

Since $\mathrm{k}>1$ and $\mathrm{B}_{1}>0$ over the operating band, the conditions of unconditional stability of the proposed BPA are met. That means that any source or load can be connected to the input or output of the BPA without risk of becoming unstable or producing oscillations.

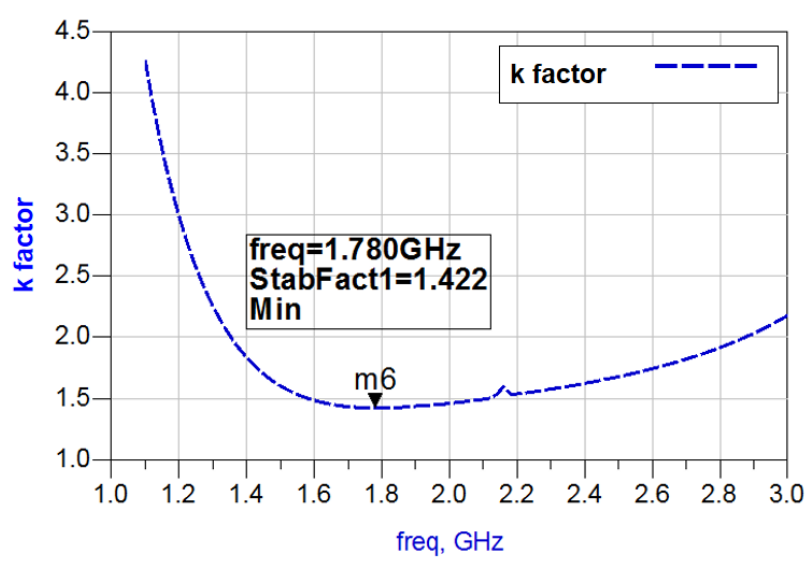

Figure.10 Curve of Rollett stability factor versus frequency

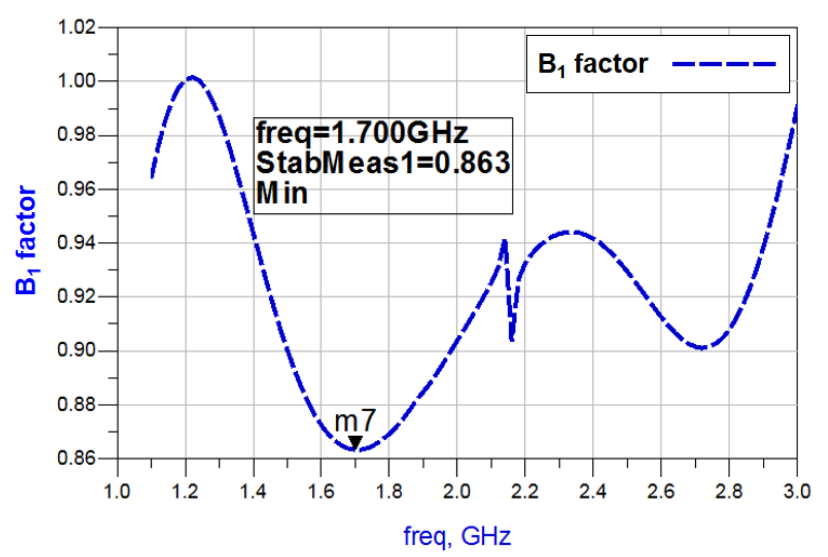

Figure. 11 Curve of Bodway stability factor B1 versus frequency

\subsection{Small signal simulation}

Due to the growing need to shorten development time, and simultaneously obtaining an accurate data, power amplifier designers are more and more use simulation tools.

The RF performance of the proposed BPA is determined using small signal, two port $\mathrm{S}$-parameter simulation. The S-parameter simulations are done in $50 \Omega$ terminations. Basic S-parameter simulations are small signal, and consists of measuring transmitted, reflected and incident waves.

Small signal gain $S_{21}$, input return loss $S_{11}$, output return loss $S_{22}$ and isolation coefficient $S_{12}$ are simulated using Advanced Design System (ADS) simulation software.

As depicted in Fig. 12, the input return loss $S_{11}$ varies between $-10.057 \mathrm{~dB}$ and $-35.948 \mathrm{~dB}$ over wide bandwidth from $1.1 \mathrm{GHz}$ to $3 \mathrm{GHz}$. Furthermore, the output return loss $\mathrm{S}_{22}$ changes between $-9.849 \mathrm{~dB}$ and $-25.631 \mathrm{~dB}$ over the prescribed band as seen in Fig. 13. It can be seen that the simulated results prove the good input and output matching over the operating 


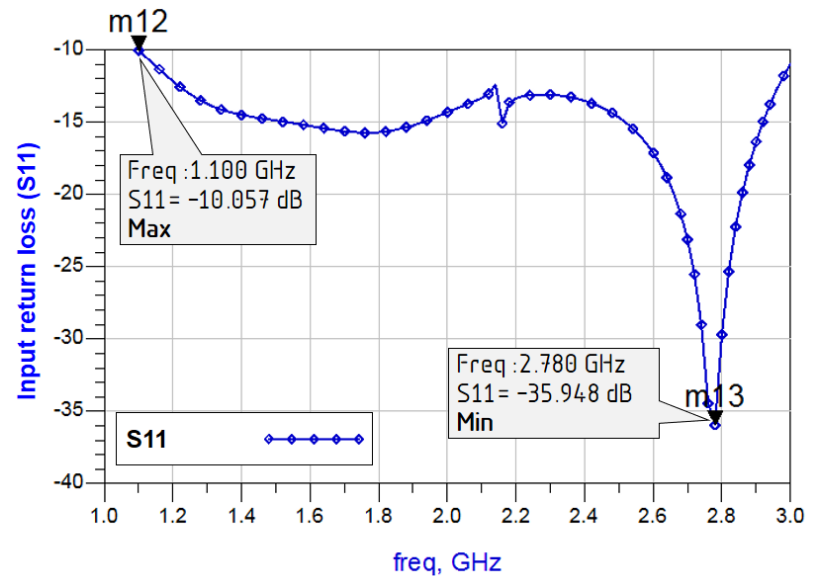

Figure. 12 Input return loss from $1.1 \mathrm{GHz}$ to $3 \mathrm{GHz}$

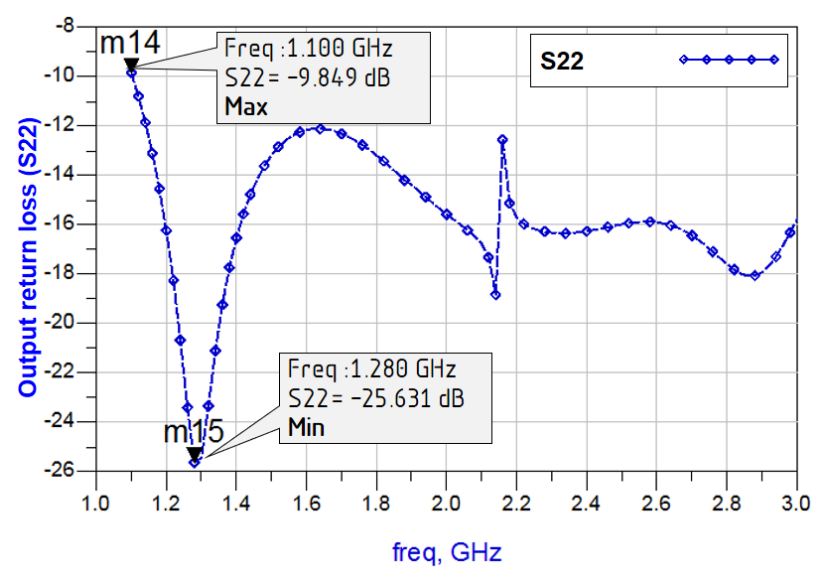

Figure. 13 Output return loss from $1.1 \mathrm{GHz}$ to $3 \mathrm{GHz}$

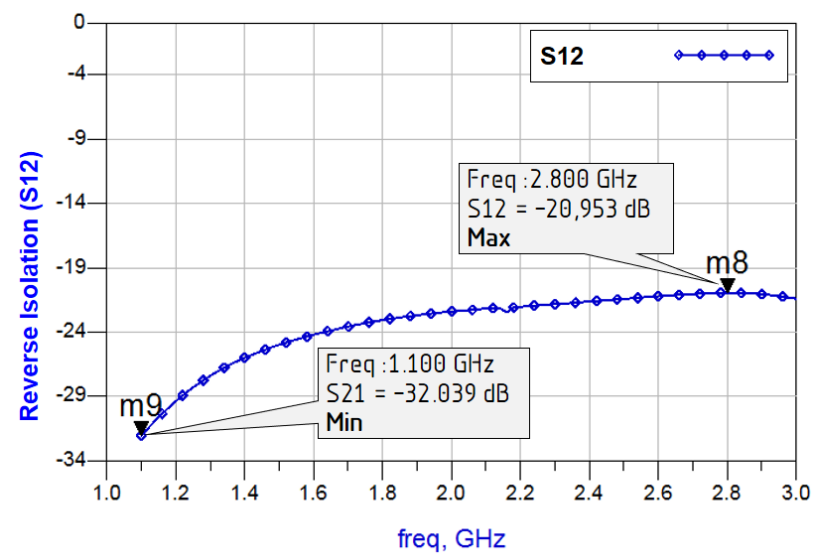

Figure. 14 Revers isolation $S_{12}$ versus frequency

bandwidth, meaning very week power is "returned" to the source from the input or to the load from the output.

The reverse isolation $S_{12}$ represents internal feedback from the input to the output of a two port device. Fig. 14 shows the simulated revers isolation of the proposed BPA. As that can be seen in Fig.14, $S_{12}$ varies between a minimum value of $-32.039 \mathrm{~dB}$ at 1.1 $\mathrm{GHz}$ and a maximum value of $-20,953 \mathrm{~dB}$ at $2.8 \mathrm{GHz}$.

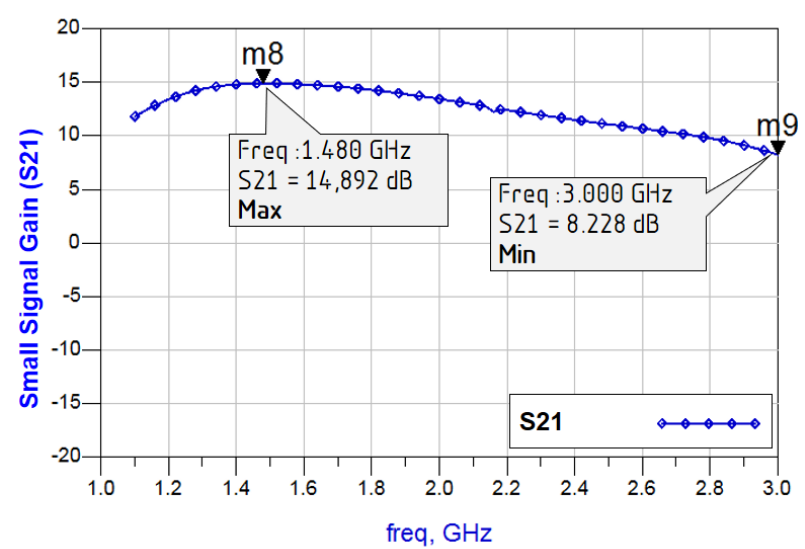

Figure. 15 Small signal gain $S_{21}$ versus frequency

In terms of operation of practical power amplifier, it is required to have $S_{12}$ as small as possible because the smaller value of reverse isolation, is the greater degree of isolation between the output and input, and also the greater degree of stability of a given stage.

The simulated gain $S_{21}$ for the proposed BPA is given in Fig. 15. As can we observe, the small-signal gain varies between a minimum value of $8.2 \mathrm{~dB}$ at $3 \mathrm{GHz}$, and a maximum value of $14.89 \mathrm{~dB}$ at 1.48 GHz.

\subsection{Large signal simulation}

In this sub-section, the simulated peak output power and power added efficiency (PAE) are presented.

As expressed by Eq. (11), the output power is the power delivered to the external load (commonly 50 $\Omega$ ) at a specify frequency or a range of frequencies.

$$
\left\{\begin{array}{c}
P_{\text {out }}=P_{\text {out }}(f)=\frac{1}{2} \mathcal{R} e\left\{V_{\text {out }} \cdot I_{\text {out }}^{*}\right\} \\
f \in\left[f_{\text {Low }} ; f_{\text {High }}\right]
\end{array}\right.
$$

The $1 \mathrm{~dB}$ compressed point is defined as the point at which the output power level corresponding a $1 \mathrm{~dB}$ deviation from the ideal linear behavior. The corresponding input power level, is used to mark the border between the linear and nonlinear regions.

From energetic point of view, a power amplifier may be eventually considered as a device converting DC power from supplies into microwave power.

The effectiveness of this conversion process is generally measured by means of Power Added Efficiency (PAE), defined as the ration between the added power and the supplied DC power:

$$
\eta_{\text {add }}=\frac{P_{a d d}}{P_{D C}}=\frac{P_{o u t}-P_{\text {in }}}{P_{D C}}
$$


Where:

$$
P_{D C}=V_{\text {bias }} \cdot \frac{1}{T} \int_{0}^{T} I_{\text {bias }}(t) \cdot d t
$$

Figs. 16 and 17 show the simulated output power and PAE of the proposed BPA at $2 \mathrm{GHz}$.

Fig. 16 shows that the simulated output power reaches a maximum of $17.14 \mathrm{dBm}$, at the $17 \mathrm{dBm}$ at the input power level, thus leading to $51.76 \mathrm{~mW}$ of output power. The proposed BPA reaches $1 \mathrm{~dB}$ compression at $4 \mathrm{dBm}$ input power level. As depicted in Fig. 17, at $2 \mathrm{GHz}$, the simulated PAE achieves a maximum value of $20.11 \%$ at $6 \mathrm{dBm}$ input power.

\section{Broadband power amplifier layout}

The layout of the proposed BPA is shown in Fig. 18 , where the input port, the output port, the bias voltage point and the active device are marked.

In this work, the proposed BPA is implemented on the substrate of Epoxy FR4, the permittivity $\left(\varepsilon_{r}\right)$ of which is 4.4. The size of the circuit plan is $10 \mathrm{~cm} \times 5 \mathrm{~cm}$. This BPA adopt the commercial transistor ATF 13786 in GaAs technology.

The performance of the designed BPA is summarized and compared to state of the art broadband power amplifiers running in $\mathrm{L}$ and $\mathrm{S}$ bands in Table 1.

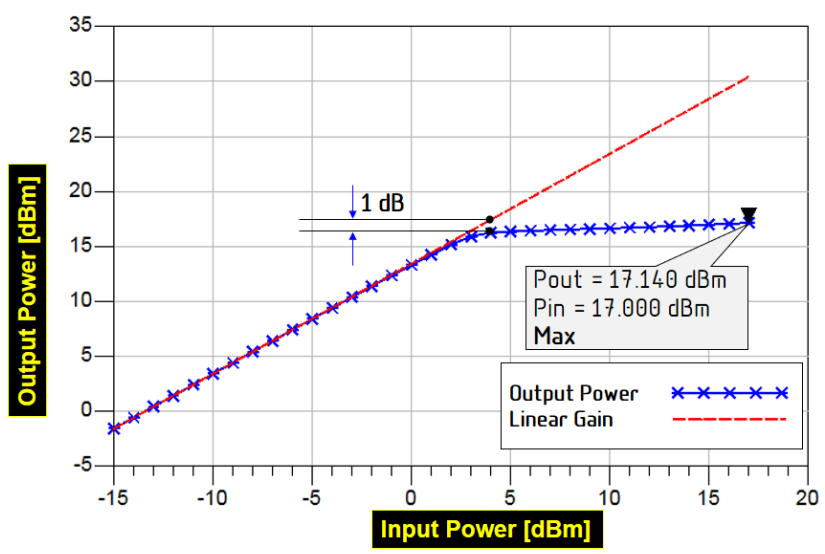

Figure.16 Output power versus input power

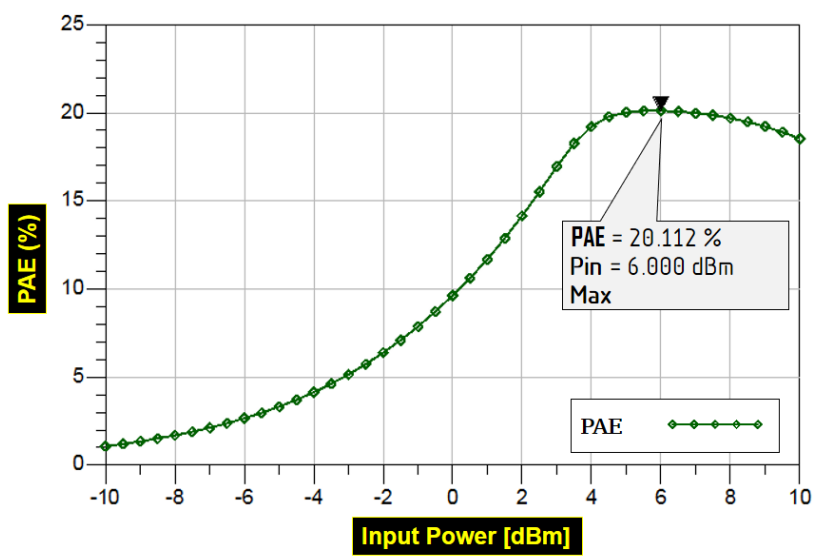

Figure.17 Power Added Efficiency versus input power

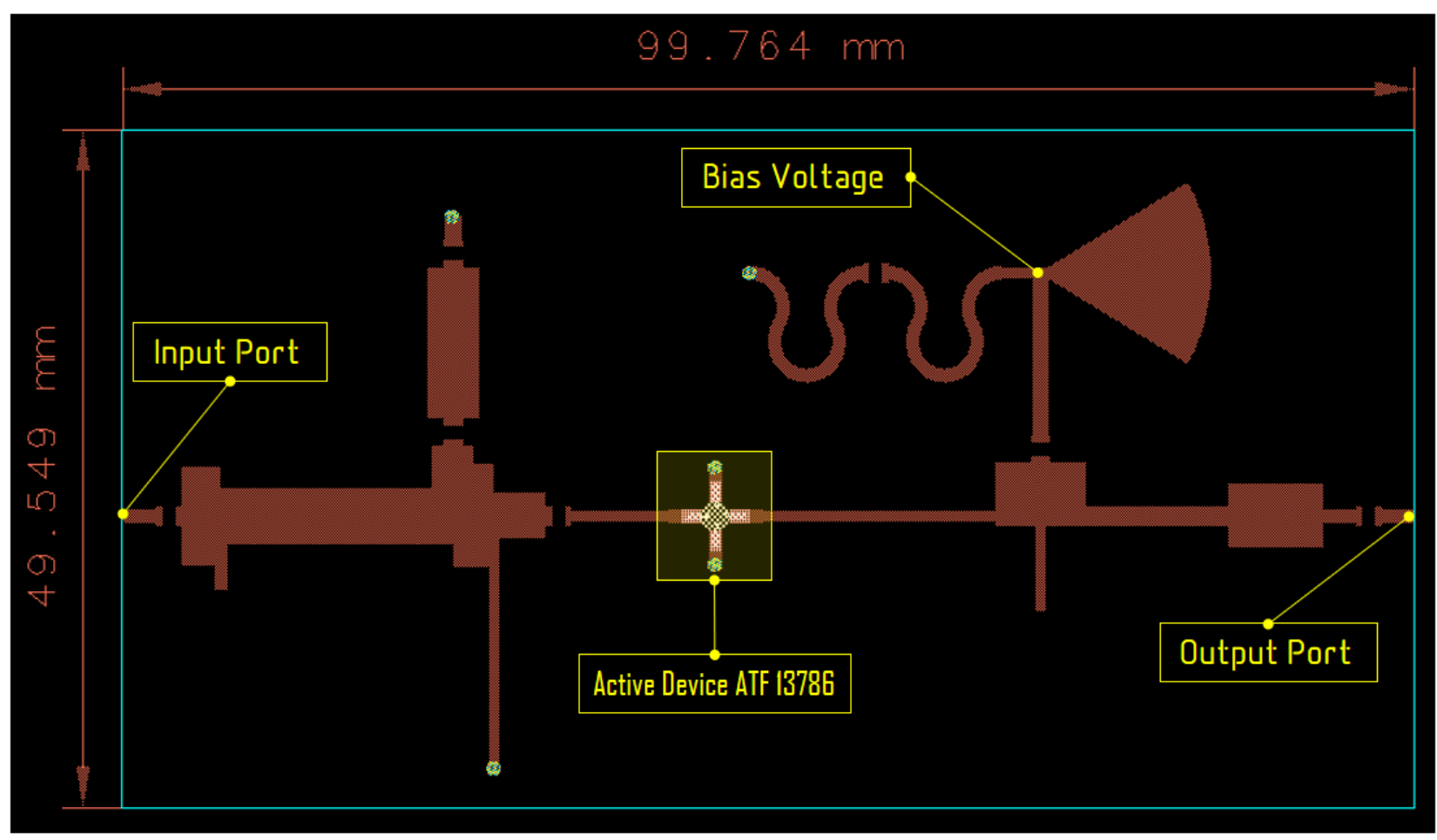

Figure.18 The proposed single stage broadband power amplifier layout 
Table 1. State of art broadband PAs

\begin{tabular}{|c|c|c|c|c|}
\hline Ref. & $\begin{array}{c}{[\mathbf{2 1}]} \\
\mathbf{2 0 1 5}\end{array}$ & $\begin{array}{c}{[\mathbf{2 2}]} \\
\mathbf{2 0 1 6}\end{array}$ & $\begin{array}{c}{[\mathbf{2 3}]} \\
\mathbf{2 0 1 8}\end{array}$ & $\begin{array}{c}\text { This } \\
\text { Work }\end{array}$ \\
\hline $\begin{array}{c}\text { Freq } \\
(\mathrm{GHz})\end{array}$ & $1.9-2.7$ & $1.8-2.8$ & $1.75-2.15$ & $1.1-3$ \\
\hline $\mathrm{P}_{\text {sat }}(\mathrm{dBm})$ & 28.1 & 28 & 8 & 17.14 \\
\hline PAE $(\%)$ & 13.7 & 6.1 & - & 20 \\
\hline Gain(dB) & 11 & 25 & $10-11.7$ & 14.9 \\
\hline S11 (dB) & - & $-12 \mid-25$ & $-11 \mid-22$ & $-10 \mid-35$ \\
\hline S22 (dB) & - & $-9 \mid-19$ & $-13 \mid-19$ & $-10 \mid-25$ \\
\hline S12 (dB) & - & - & $-18 \mid-19$ & $-20 \mid-32$ \\
\hline $\begin{array}{c}\text { Supply } \\
(\mathrm{V})\end{array}$ & 2.5 & 5 & 12 & 3 \\
\hline
\end{tabular}

Comparing the present work with similar contemporary state-of-art broadband PAs [21-23], we can clearly observe that the proposed broadband matching techniques are useful, and the designed BPA reaches an excellent bandwidth with good matching across the operating frequency ranges.

The simulated return loss does show good results over $2900 \mathrm{MHz}$ of bandwidth compared to [21 - 23].

In the frequency range of $1.1 \mathrm{GHz}$ to $3 \mathrm{GHz}$, the proposed BPA achieves a maximum gain of $14.9 \mathrm{~dB}$, and a $\mathrm{P}_{\text {sat }}$ of $17.14 \mathrm{dBm}$ with an $20 \% \mathrm{PAE}_{\max }$ and a $\mathrm{P}_{1 \mathrm{~dB}}$ of $4 \mathrm{dBm}$ under the $3 \mathrm{~V}$ supply voltage.

Considering the wideband input power and the broadband frequency coverage, the proposed BPA achieves better wideband large-signal performances compared with other BPAs [21 - 23], and exhibits high gain, excellent bandwidth, good output power, good matching across operating bandwidth, and simple circuitry. It is a good candidate for the broadband PAs operates in $\mathrm{L}$ and $\mathrm{S}$ bands.

\section{Conclusion}

In this paper, a broadband power amplifier used for $\mathrm{L}$ and $\mathrm{S}$ bands applications has been presented. Realized in GaAs process, the proposed BPA achieves a maximum gain of $14.89 \mathrm{~dB}$ in the whole frequency range of $1.1 \sim 3 \mathrm{GHz}$.

With neatly designed matching networks and bias circuit, good input and output matching is reached over a broad frequency range, and the return loss varies between $-10 \mathrm{~dB}$ and $-35 \mathrm{~dB}$, the output return loss between $-10 \mathrm{~dB}$ and $-25 \mathrm{~dB}$, and the reverse isolation between $-20.9 \mathrm{~dB}$ and $-32 \mathrm{~dB}$.

At $2 \mathrm{GHz}$, the large signal simulation shows that the proposed BPA exhibits the saturated output power of $17.14 \mathrm{dBm}$, output $1-\mathrm{dB}$ compressed point of $4 \mathrm{dBm}$ input power level, and PAE of $20 \%$.

The whole circuit is unconditionally stable over the full bandwidth from $1.1 \mathrm{GHz}$ to $3 \mathrm{GHz}$. A power amplifier with these performances characteristics is suitable for many applications operating in $\mathrm{L}$ and $\mathrm{S}$ bands such as SRAN (Single Radio Access Network) in cellular communications, avionics, medical microwave imaging, electronic warfare, and digital television broadcasting represent just a few examples.

\section{References}

[1] I. J. Bahl, Fundamentals of RF and Microwave Transistor Amplifiers, John Wiley \& Sons, Inc, Hoboken, New Jersey, 2009.

[2] M. Bozanic, S. Sinha, Power Amplifier for the $S$-, $C$-, $X$ - and $k u$ bands, Springer International, Switzerland, 2016.

[3] IEEE Std 521-1984, IEEE Standard Letter Designations for Radar-Frequency Bands, 1984.

[4] M. K. Kazimierczuk, RF Power Amplifiers, $2^{\text {nd }}$ edition, John Wiley \& Sons, Inc, 2015.

[5] M. Ribate, R. Mandry, M. Latrcach, A. Errkik, and A. El Abdelaoui, "GaAs FET Broadband Power Amplifier for $\mathrm{L}$ and $\mathrm{S}$ Bands Applications", In: Proc. of the $2^{\text {nd }}$ International Conf. On Computing and Wireless Communication Systems, 2017.

[6] J. M. Osepchuck, "Microwave Power Applications", IEEE Transactions on Microwave Theory and Techniques, Vol.50, No.3, pp.975985, 2002.

[7] I. T. Rekanos, A. Räisänen, "Microwave Imaging in the Time Domain of Buried Multiple Scatterers by Using an FDTD-Based Optimization Technique", IEEE Transactions on Magnetics, Vol.39, No.3, pp. 1381-1384, 2003.

[8] A. Rosen, M. A. Stuchly, and A. V. Vorst, "Applications of RF/Microwaves in Medicine", IEEE Transactions on Microwave Theory and Techniques, Vol.50, No.3, pp.963-974, 2002.

[9] J. Walker, Handbook of RF and Microwave Power Amplifiers, Cambridge University press, 2012.

[10] B. Liu, M. Mao, D. Khanna, C. Boon, P. Choi, and E.A. Fitzgerald, "A Novel 2.6-6.4 GHz Highly Integrated Broadband $\mathrm{GaN}$ Power Amplifier", IEEE Microwave and Wireless Components Letters, Vol.28, No.1, pp.37-39, 2018.

[11] M. S. Khan, H. Zhang, X. Wang, R. Ullah, I. Ahmed, S. Shahzad, Q. A. Arain, and M. Z. Tunio, "A Novel Two-Stage Broadband Doherty Power Amplifier for Wireless Applications", IEEE Microwave and Wireless Components Letters, Vol.28, No.1, pp.40-42, 2018.

[12] D. Liu and Q. F. Cheng, "A 4 GHz HighEfficiency High-Linearity GaN Doherty Power 
Amplifier with Dual DC-Power Supplies", International Journal of Intelligent Engineering and Systems, Vol.8, No.3, pp.123-456, 2015.

[13] D.W. Kim, "An Output Matching Technique for a GaN Distributed Power Amplifier MMIC Using Tapered Drain Shunt Capacitors", IEEE Microwave and Wireless Components Letters, Vol.25, No.9, pp.603-605, 2015.

[14] A. Alizadeh and A. Medi, "Distributed Class-J Power Amplifiers", IEEE Transactions on Microwave Theory and Techniques, Vol. 65, No. 2, pp. 512-521, 2016.

[15] M. Li, "A Millimeter Wave Broadband GaAs Power Amplifier with Balanced Bias Feedings for Stability Enhancement", In: Proc. of the 17th IEEE Annual Wireless and Microwave Technology Conference, 2016.

[16] K. Chen and D. Peroulis, "Design of Highly Efficient Broadband Class-E Power Amplifier Using Synthesized Low-Pass Matching Networks", IEEE Transactions on Microwave Theory and Techniques, Vol. 59, No.12, pp. 3162-3173, 2011.

[17] C. Poole and I. Darwazeh, Microwave Active Circuit Analysis and Design, Elsevier Ltd, 2016.

[18] N. Kumar and A. Grebennikov, Distributed Power Amplifiers for $R F$ and Microwave Communications, Artech House, 2015.

[19] K. W. Yeom, Microwave Circuit Design, a Practical Approach Using ADS, Prentice Hall, 2015.

[20] I. Bahl and P. Bhartia, Microwave Solid State Circuit Design, John Wiley \& Sons, Inc, New Jersey, 2003.

[21] B. Francois and P. Reynaert, "Highly linear fully integrated wideband RF PA for LTE-advanced in 180-nm SOI", IEEE Transactions on Microwave Theory and Techniques, Vol. 63, No.3, pp. 649-658, 2015.

[22] C.Q. Chen, M.L. Hao, Z.Q. Li, Z.B. Du, and H. Yang, "A $1.8-2.8 \mathrm{GHz}$ Highly Linear Broadband Power Amplifier for LTE-A Application", Progress in Electromagnetics Research C, Vol. 66, pp.47-54, 2016.

[23] A. Rachakh, L. El Abdellaoui, J. Zbitou, A. Errkik, A. Tajouati, and M. Latrach, "A Novel Configuration of a Microstrip Microwave Wideband Power Amplifier for Wireless Application", Telkomnika, Vol.16, No.1, pp.2014-2031, 2018.

[24] P. Jarry and J.N. Beneat, Microwave Amplifier and Active Device Design Using the Real Frequency Technique, John Wiley \& Sons, Inc, Hoboken, New Jersey, 2016. 